\title{
First-principles description of atomic gold chains on $\mathrm{Ge}(001)$
}

\author{
S. López-Moreno, ${ }^{1}$ A. H. Romero, ${ }^{1}$ A. Muñoz, ${ }^{2}$ and U. Schwingenschlögl ${ }^{3, *}$ \\ ${ }^{1}$ CINVESTAV-Queretaro, Libramiento Norponiente No. 2000, Real de Juriquilla, 76230 Queretaro, Mexico \\ ${ }^{2}$ MALTA Consolider Team, Departamento de Física Fundamental II, Instituto de Materiales y Nanotecnología, \\ Universidad de La Laguna, La Laguna 38205, Tenerife, Spain \\ ${ }^{3}$ PSE Division, KAUST, Thuwal 23955-6900, Saudi Arabia
}

(Received 9 August 2009; revised manuscript received 31 October 2009; published 25 January 2010)

\begin{abstract}
We have performed density-functional theory calculations, including the spin-orbit correction, to investigate atomic gold chains on $\mathrm{Ge}(001)$. A set of 26 possible configurations of the $\mathrm{Au} / \mathrm{Ge}(001)$ system with $c(4 \times 2)$ and $c(8 \times 2)$ symmetries is studied. Our data show that the $c(4 \times 2)$ order results in the lowest energy, which is not in direct agreement with recent experiments. Using total-energy calculations, we are able to explain these differences. We address the electronic band structure and apply the Tersoff-Hamann approach to correlate our data to scanning-tunneling microscopy (STM). We obtain two highly competitive structures of the atomic Au chains for which we report simulated STM images in order to clarify the composition of the experimental $\mathrm{Au} / \mathrm{Ge}(001)$ surface.
\end{abstract}

DOI: 10.1103/PhysRevB.81.041415

Having been investigated steadily since decades, semiconductor surfaces are recently attracting new attention due to a great capacity of hosting monatomic metallic chains. While mechanical fabrication by scanning-tunneling microscopy (STM) tip techniques ${ }^{1,2}$ or break junction techniques ${ }^{3,4}$ is limited to a chain length of several ten atoms, utilization of self-organization processes of adatoms on surfaces can lead to much longer chains. These chains are of great interest both for basic research and for various applications because they give rise to a (quasi-) one-dimensional electron system. In this context, Schäfer et al. ${ }^{5}$ recently discovered a new system: self-organized atomic $\mathrm{Au}$ chains on $\mathrm{Ge}(001)$ with a $c(8 \times 2)$ long-range order. These Au nanowires appear to be largely decoupled from the Ge substrate and therefore have been put forward as a model system for a Luttinger liquid. ${ }^{6-8}$

Self-organized In wires on Si(111) (Ref. 9) and Au wires on $\mathrm{Si}(533),{ }^{10}$ on the contrary, have been found to exhibit a significant coupling to the substrate. For the Ge(001) surface, $\mathrm{Au}$ growth, in general, comes along with a large variety of ordering phenomena as a function of both coverage and growth temperature. ${ }^{11}$ The $\mathrm{Ge}(001)$ surface thereby is subject to a strong relaxation. In particular, a missing-row reconstruction with emerging microfacets of the Ge(111) type, ${ }^{12}$ which is known from the clean $\mathrm{Au}(110), \operatorname{Ir}(110)$, and $\operatorname{Pt}(110)$ surfaces, ${ }^{13,14}$ has been reported. Adsorption of Pt atoms on $\mathrm{Ge}(001)$ results in highly ordered arrays of nanowires, which show very little defects and reach lengths of several 100 $\mathrm{nm} .^{15,16}$ The latter system has been investigated in detail ${ }^{17,18}$ by $a b$ initio calculations based on the density-functional theory (DFT), ${ }^{19}$ demonstrating the capacity of $a b$ initio methods for interpreting experimental data, particularly, from STM and angular-resolved photoelectron spectroscopy. Specifically, the pseudopotential method provides useful information by accurate theoretical STM images, which help to understand experimental data. ${ }^{20}$

Our findings are based on total-energy DFT calculations in the framework of the projector-augmented wave method, ${ }^{21,22}$ using the Vienna ab initio simulation package. ${ }^{23,24}$ For the exchange-correlation functional, we apply the generalized gradient approximation and the PerdewBurke-Ernzerhof scheme. ${ }^{25}$ All our data have been obtained
PACS number(s): 73.20.-r, 62.23.Hj, 73.21.Hb, 73.63.Nm

under the inclusion of the spin-orbit coupling, which is essential for describing the electronic as well as optical properties of $\mathrm{Au}$ systems. ${ }^{26} \mathrm{We}$ use a 250 -eV-plane-wave energy cutoff and have carefully checked the convergence of our calculations. The Monkhorst scheme is employed for Brillouin-zone integrations, ${ }^{27}$ applying meshes of $10 \times 10$ $\times 10$ and $8 \times 4 \times 1$ points in the bulk and the surface calculation, respectively. Our structure optimization leads to residual forces of less than $0.01 \mathrm{eV} / \AA$ (for each Cartesian direction).

Our calculations provide key results on the behavior of self-assembled $\mathrm{Au}$ atoms on a $\mathrm{Ge}(001)$ surface and on the question of how the ordering is affected by the presence of the substrate. The interaction is not only due to electron transfer but is also determined by a reconstruction on an atomic scale, which draws adatoms into the surface.

A good overview of the experimental and theoretical efforts to resolve the surface reconstruction of the clean $\mathrm{Ge}(001)$ surface can be obtained from Refs. 28 and 29. It has been established that neighboring Ge surface atoms form asymmetric dimers in order to saturate one dangling bond, whereas the relaxation of the bulk is rather small. The dimers line up to characteristic dimer rows. Moreover, the lattice is further stabilized by a distinct buckling pattern of the surface dimers, which leads to a $c(4 \times 2)$ superstructure at low temperatures. DFT-based structure optimization has fully reproduced $\mathrm{x}$-ray diffraction data for the clean $\mathrm{Ge}(001)$ surface $^{30,31}$ and, in addition, showed a good agreement for the resulting surface electronic states. ${ }^{32}$ We simulate the $\mathrm{Ge}(001)$ surface by means of repeated asymmetric slabs of eight Ge layers and a vacuum region of the same size. The top five Ge layers are allowed to relax and the bottom side of each slab (with fixed atomic positions) is saturated by $\mathrm{H}$ atoms in order to simulate the continuation of the Ge bulk.

We calculate the ground-state total energy for $c(8 \times 2)$ as well as $c(4 \times 2)$ coverages with 26 configurations in total. Structures are built for Au atoms on substitutional and adsorption sites. In each case, we find the lowest energy of the $c(4 \times 2)$ coverage at least $0.8 \mathrm{eV}$ below that of the $c(8 \times 2)$ coverage. Subsequently, we thus focus on the $c(4 \times 2)$ coverage, for which we consider 11 configurations. Zero-point 


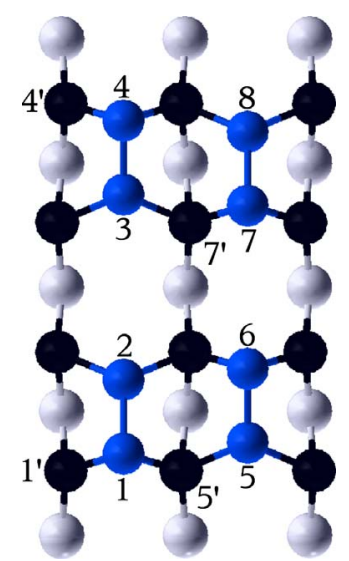

(a)

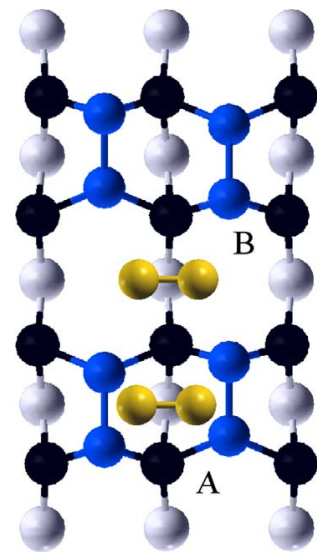

(b)
FIG. 1. (Color online) (a) Relaxed clean Ge(001) surface with the most likely Au positions for a coverage of 1/4 ML. Labels with tilde correspond to atoms in the second layer. (b) Adsorption sites of Au surface dimers. Ge surface atoms, second layer atoms, and third layer atoms are shown in blue (dark gray), black, and light gray, respectively.

energies are not included. We substitute two atoms of the $\mathrm{Ge}(001)$ surface by Au [see Fig. 1(a)] and account for the adsorbed Au dimers shown in Fig. 1(b). The surface formation energy per unit cell is given by

$$
\Omega_{f}=E_{\mathrm{Au} / \mathrm{Ge}(001)}-\mu_{\mathrm{Ge}} N_{\mathrm{Ge}}-\mu_{\mathrm{Au}} N_{\mathrm{Au}}-\mu_{\mathrm{H}} N_{\mathrm{H}},
$$

where $E_{\mathrm{Au} / \mathrm{Ge}(001)}$ is the total energy of a slab containing $N_{\mathrm{Ge}}$ $\left(N_{\mathrm{Au}}, N_{\mathrm{H}}\right) \mathrm{Ge}(\mathrm{Au}, \mathrm{H})$ atoms. The corresponding chemical potentials are represented by $\mu_{\mathrm{Ge}}\left(\mu_{\mathrm{Au}}, \mu_{\mathrm{H}}\right)$. Since Eq. (1) gives the formation energy of both surfaces of the slab, and we are only interested in the surface with the adsorbed Au atoms, we only study the relative formation energy of the Au-covered system

$$
\Delta \Omega_{f}=E_{\mathrm{Au} / \mathrm{Ge}(001)}-E_{\mathrm{Au} / \mathrm{Ge}(001)}^{r e f}-\mu_{\mathrm{Ge}}\left(N_{\mathrm{Ge}}-N_{\mathrm{Ge}}^{0}\right),
$$

with respect to a reference adsorbate $E_{\mathrm{Au} / \mathrm{Ge}(001)}^{r e f}$.

The 13 configurations reported in this work are labeled according to their Au positions on the $\mathrm{Ge}(001)$ surface [see Figs. 1(a) and 1(b)]. The relative surface energies are calculated by Eq. (2) (see the summary in Table I, where the 2-8 model is the reference system). By the spin-orbit correction, the energies of the various configurations are modified and also new features appear in the electronic dispersion: shifts of energy levels, reflecting a spin-orbit splitting and "kinks" in the band structure, compare the later discussion. In the case of Au dimers, models $\mathrm{A}$ and $\mathrm{B}$, the relaxation breaks up the $\mathrm{Au}-\mathrm{Au}$ dimer bond and a single $\mathrm{Au}$ atom is adsorbed. In model $\mathrm{A}$, each $\mathrm{Au}$ atom bonds to the nearest $\mathrm{Ge}$ dimer atom by breaking up the buckled dimer, while the next dimer row is not affected. However, in model B, the bond length of the 4 Ge surface dimers increases from $2.58 \AA$ (see the top view in Fig. 2) to 2.68, 2.75, 2.87, and $2.66 \AA$.

The 1-6 and 2-5 models show a zig-zag order of Au-Ge dimers in a single row, while the next row is formed by $\mathrm{Ge}-\mathrm{Ge}$ dimers. The relative surface energy difference is very small. A reason could be that in the $2-5$ model, the Ge-Ge/
TABLE I. Relative surface formation energy as defined from Eq. (2), in $\mathrm{meV} / 1 \times 1$ unit cell, for the $\mathrm{Au} / \mathrm{Ge}(001)$ adsorbate system. Fixed $\mathrm{Au}$ number $N_{\mathrm{Au}}=2$ and $\mathrm{H}$ number $N_{\mathrm{H}}=16$, per asymmetric slab. The number of Ge atoms $\left(N_{\mathrm{Ge}}\right)$ varies. For $\Delta \Omega_{f}$, the 2-8 model is used as reference system. Values in brackets are obtained without spin-orbit interaction. Beyond, $\mu_{\mathrm{Ge}}=-4.50 \mathrm{eV}, \mu_{\mathrm{Au}}$ $=-3.60 \mathrm{eV}$, and $\mu_{\mathrm{H}}=-3.35 \mathrm{eV}$.

\begin{tabular}{lccccccc}
\hline \hline Model & $N_{\mathrm{Ge}}$ & $\Delta \Omega_{f}$ & & Model & $N_{\mathrm{Ge}}$ & $\Delta \Omega_{f}$ & \\
\hline A & 64 & 871 & $(839)$ & $2-5$ & 62 & 74 & $(81)$ \\
$\mathrm{B}$ & 64 & 859 & $(816)$ & $1-8$ & 62 & 191 & $(211)$ \\
$4^{\prime}-7$ & 62 & 595 & $(668)$ & $2-8$ & 62 & 0 & $(0)$ \\
$1-5$ & 62 & 20 & $(49)$ & $1-7^{\prime}$ & 62 & 690 & $(764)$ \\
$1-6$ & 62 & 90 & $(96)$ & $2-7^{\prime}$ & 62 & 459 & $(508)$ \\
$1-7$ & 62 & 382 & $(421)$ & $2-4^{\prime}$ & 62 & 461 & $(511)$ \\
$1^{\prime}-5^{\prime}$ & 62 & 649 & $(751)$ & & & & \\
\hline \hline
\end{tabular}

Au-Ge dimers maintain the asymmetric order of the clean $\mathrm{Ge}(001)$ surface, as it is illustrated in Fig. 2. In contrast, in the 1-6 model, the asymmetric dimer order in the [110] direction is broken, while a zig-zag order along the [001] direction is maintained. The $1-7$ model is very closely related to the experimental structure, ${ }^{5}$ despite its $c(4 \times 2)$ coverage.
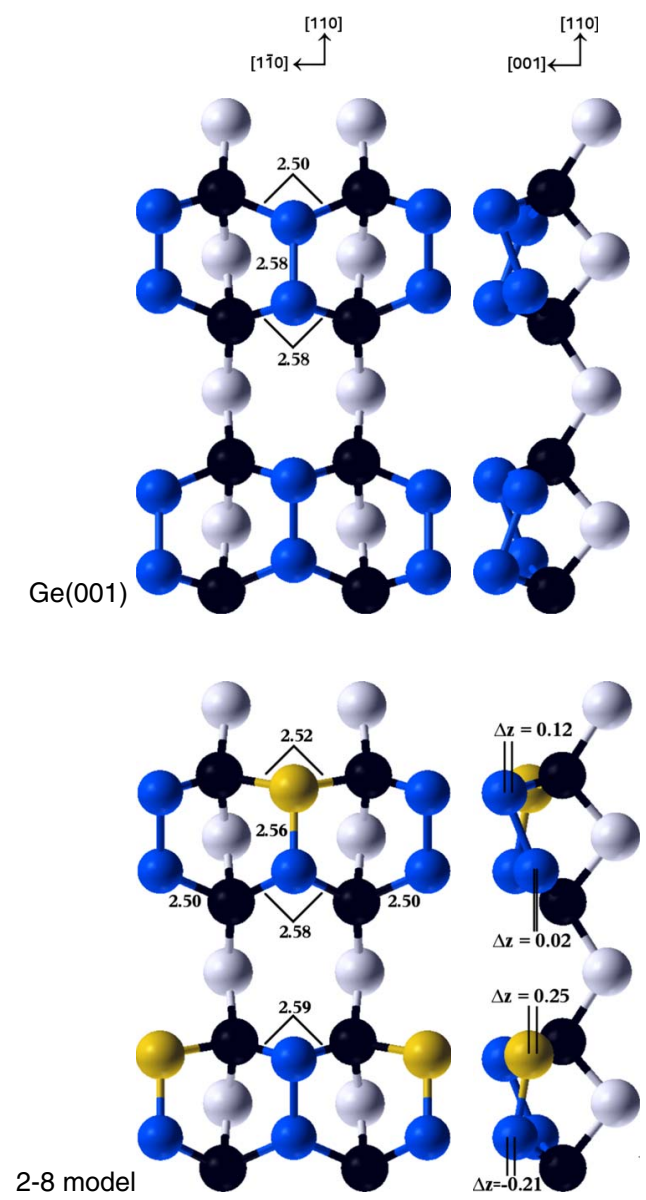

FIG. 2. (Color online) Top and side views of the relaxed clean $\mathrm{Ge}(001)$ surface and the $2-8$ model. $\Delta z$ denotes the shift of the dimer atoms with respect to the clean surface. All values are given in $\AA$. 


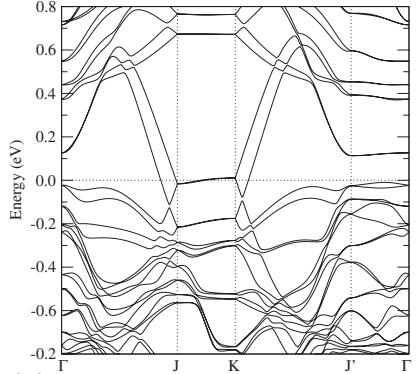

(a)

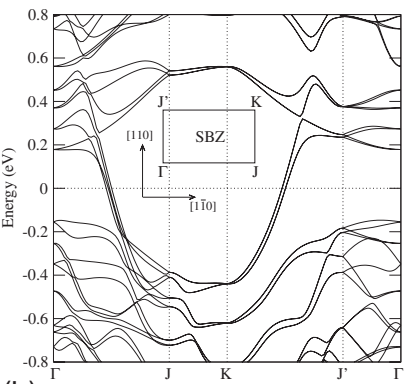

(b)

FIG. 3. Electronic band structures for the models (a) 1-5 and (b) $2-8$. The zero energy is given by the Fermi level.

The Au atoms occupy upper dimer sites and are strongly bound to the second Ge layer and the lower Ge dimer atom. A structure optimization leads to an essentially flat surface similar to nonrelaxed $\mathrm{Ge}(001)$ with two dangling bonds. Thus, both the zig zag and the asymmetric order of the dimers disappear.

In the 1-8 model, the Au atoms occupy one upper and one lower dimer sites. The first $\mathrm{Au}$ atom breaks the asymmetric order of the buckled dimers (similar to 1-7), while the second does not affect the dimer (similar to 2-8). As a consequence, the 1-8 model lies, energetically, between the 1-7 and 2-8 models, in which the gold atoms have a larger distance to each other. The second lowest relative surface energy is found for the 1-5 model. Here, the Au atoms are arranged as chains running in [1 $\overline{1} 0]$ direction, alternately occupying upper and lower dimer sites. Each Au chain is separated from the next chain by almost $16 \AA$, which is exactly the reported experimental value. We observe that the $\mathrm{Au}-\mathrm{Ge}$ dimers again form a flat surface, while the Ge-Ge dimers maintain the asymmetric order. The $z$ coordinate of a Ge-Ge dimer's center of mass deviates from the respective value for an Au-Ge dimer by only $0.04 \AA$. Unlike the $1-7$ and 1-8 models in which the horizontal dimer order increases the surface energy, here an increase is compensated by the $\mathrm{Au}-\mathrm{Au}$ interaction.

The lowest-energy configuration is the $2-8$ model, with a $c(4 \times 2)$ order, as seen in Fig. 1 . In this case, the dimers are ordered asymmetrically, as in the clean Ge(001) surface. Our results show a tendency to preserve the bond distances of the $\mathrm{Au}-\mathrm{Ge}$ dimer, both in the Ge-Ge dimers and on the second Ge layer. A comparison between the 2-8 model and the relaxed clean $\mathrm{Ge}(001)$ surface is shown in Fig. 2, including several dimer bond lengths. The values illustrate the modification due to the introduction of $\mathrm{Au}$ atoms. $\Delta z$ quantifies the shifts of the dimer atoms with respect to the clean surface.

In order to identify the effects of the Au-Au bonding and the interplay with the Ge surface, we turn to the electronic band structure shown in Fig. 3 for the 1-5 and 2-8 models along selected high-symmetry lines of the surface Brillouin zone. The energy zero is defined by the Fermi level. Because the bands above $0.5 \mathrm{eV}$ and below $-0.5 \mathrm{eV}$ mainly represent states of the clean $\mathrm{Ge}(001)$ surface, we focus on the energy range $\pm 0.5 \mathrm{eV}$ around the Fermi energy, which is influenced by the Au-Ge interplay. For the 1-5 model, the bands in this energy range resemble those of the tetradimer chain (TDC) model for the $\mathrm{Pt} / \mathrm{Ge}(001)$ system. ${ }^{33}$ In the TDC model, the Pt

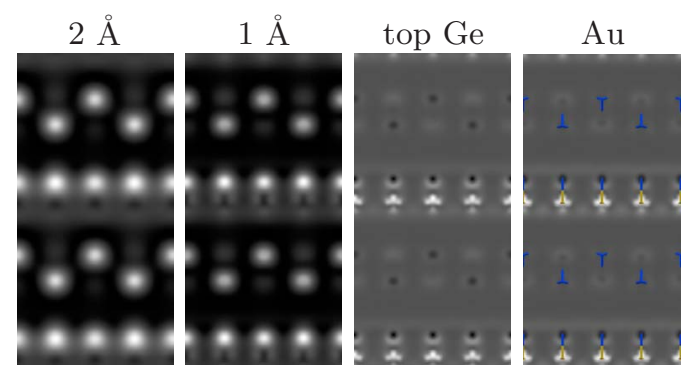

FIG. 4. (Color online) STM images of a $2 \times 2$ supercell simulated for the $1-5$ model at $-0.2 \mathrm{~V}$. The constant-height images are calculated for planes 2, 1 , and $0 \AA$ above the topmost Ge atoms and at the $\mathrm{Au}$ atoms. Au panel: $\mathrm{Ge}$ and $\mathrm{Au}$ atoms are marked blue (dark gray) and yellow (light gray), respectively.

atoms likewise replace Ge dimer atoms, but there is another Ge-Ge dimer in the B position of Fig. 1(b), bound to the Pt atoms and the Ge atoms in the next dimer row.

According to Fig. 3, the bands around the Fermi level show large dispersions along $\Gamma-J$ and $K-J^{\prime}$, i.e., along the $\mathrm{Au}$ chains in the $[1 \overline{1} 0]$ direction. On the other hand, there is

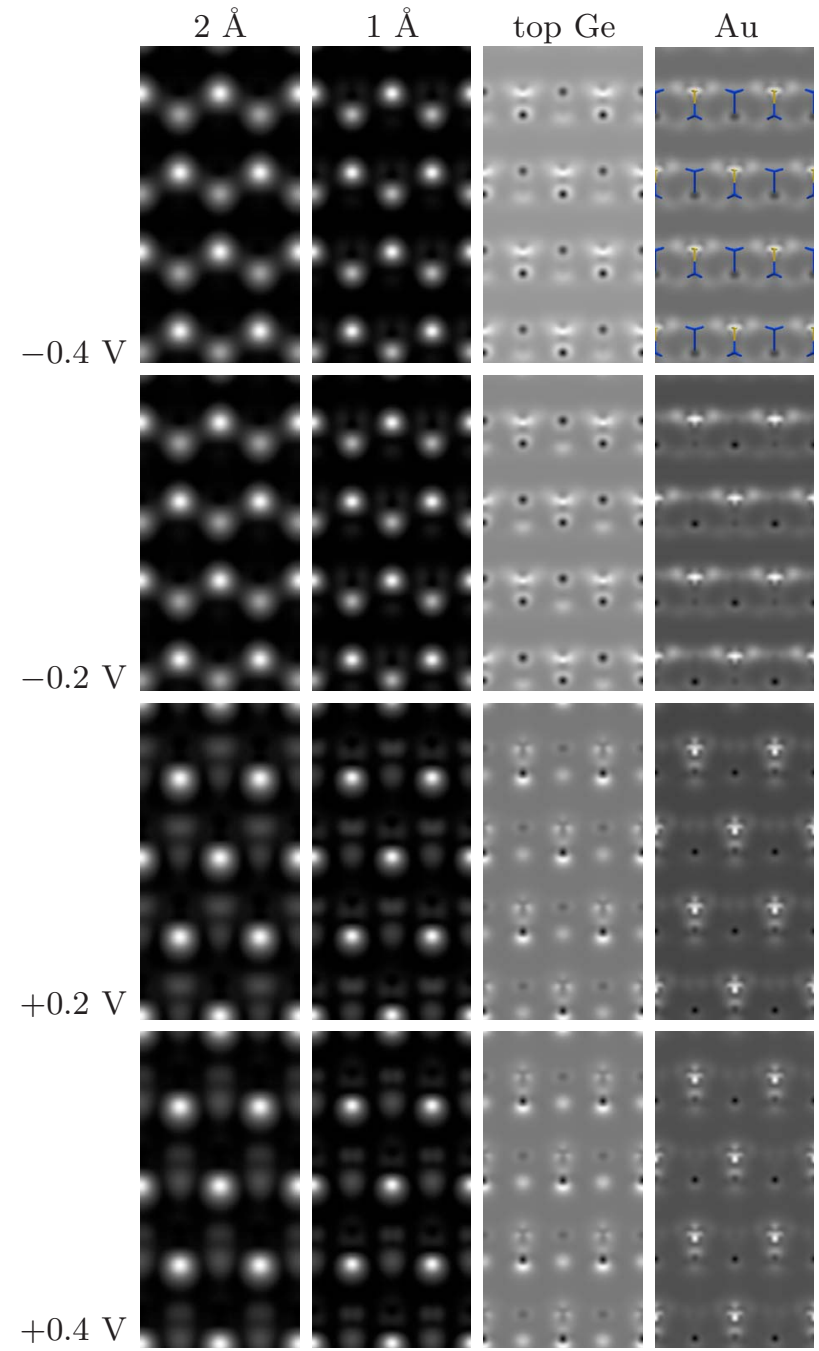

FIG. 5. (Color online) STM images of a $2 \times 2$ supercell simulated for the $2-8$ model between -0.4 and $+0.4 \mathrm{~V}$, analogous to Fig. 4. Positive voltages refer to unoccupied states. 
much less dispersion along $J-K$ and $J^{\prime}-\Gamma$, i.e., perpendicular to the chains. By increasing the fineness of the $k$ sampling, we have verified that the "kinks" visible in the band structures are no artifacts. A band crossing at these points definitely can be excluded by an analysis of the eigenvectors corresponding to the nearby bands.

Different dispersions along the high-symmetry lines of the Brillouin zone are confirmed by the STM images in Fig. 4. These constant-height images are calculated for a voltage of $-0.2 \mathrm{~V}$ and planes 2,1 , and $0 \AA$ above the topmost surface $\mathrm{Ge}$ atoms. Moreover, we study a plane just above the $\mathrm{Au}$ atoms. Figure 4 illustrates the formation of an $\mathrm{Au}$ chain in

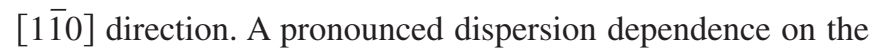
wire direction has also been obtained experimentally. ${ }^{5}$

The bands are more dispersive along $\Gamma-J$ and $K-J^{\prime}$ for the 2-8 model, as compared to the $1-5$ model, which is explained by the alignment of the $\mathrm{Au}$ atoms along the [1 $\overline{1} 0]$ direction. Because of the smaller distance between the Au atoms in the $[1 \overline{10}]$ than in the [110] direction, the dispersive character of the bands is increased. The dispersion along $\Gamma-J$ strongly distinguishes the 2-8 model from the 1-5 model. The bands behave similar to those of the clean $\mathrm{Ge}(001)$ surface because the 2-8 model maintains the asymmetric dimers. In contrast, the 1-5 model has both a row of horizontal $\mathrm{Au}-\mathrm{Ge}$ dimers in the $[1 \overline{1} 0]$ direction and a row of asymmetric $\mathrm{Ge}-\mathrm{Ge}$ dimers. This fact is reflected by the simulated STM images for the 1-5 model in Fig. 4. We find a linear structure of horizontal Au-Ge dimers and a zig-zag structure of Ge-Ge dimers. For the 2-8 model, the simulated STM images in Fig. 5 show zig-zag chains, as known from clean $\mathrm{Ge}(001)$. In the Au layer, we find in the occupied range $(-0.4 \mathrm{~V},-0.2 \mathrm{~V})$ the (one dimensional) delocalized nanowire states. It has been pointed out in Ref. 5 that STM data are not able to clarify the atomic composition of the $\mathrm{Au} / \mathrm{Ge}(001)$ surface due to atomic localization effects. According to our calculations, this fact is rather due to the reconstruction of the $\mathrm{Au}$ atoms on the surface. Because our data show that the metallic wires are formed below the topmost Ge layer, an observation by STM will be difficult.

To conclude, we have investigated the formation of $\mathrm{Au}$ chains on the reconstructed $\mathrm{Ge}(100)$ surface by means of density-functional theory. We show that it is important to include spin-orbit effects to properly describe the surface energies and the electronic structure. We also find a good agreement between our calculations and previously reported experiments. Our ground-state solutions reveal two competitive structures with a similar surface energy but different reconstruction patterns. In the first, the Au atoms sit on top of the Ge surface, whereas in the second they are located below the topmost Ge layer. STM simulations indicate that the Au chains can be detected only in a very narrow voltage range. Our results demonstrate that they are almost perfectly one dimensional with very little dispersion in the perpendicular direction.

A.M. acknowledges financial support of the MICINN of Spain under Grants No. MAT2007-65990-C03-03 and No. CSD2007-00045 and the computer resources provided by MareNostrum. A.H.R. and S.L.-M. have been supported by CONACyT Mexico under Project No. J-59853-F and by the PROALMEX/DAAD. Further computer resources have been provided by CNS IPICYT Mexico.

*udo.schwingenschlog1@kaust.edu.sa

${ }^{1}$ N. Nilius, T. M. Wallis, and W. Ho, Science 297, 1853 (2002).

${ }^{2}$ J. Lagoute, X. Liu, and S. Fölsch, Phys. Rev. B 74, 125410 (2006).

${ }^{3}$ A. I. Yanson et al., Nature (London) 395, 783 (1998).

${ }^{4}$ S. R. Bahn and K. W. Jacobsen, Phys. Rev. Lett. 87, 266101 (2001).

${ }^{5}$ J. Schäfer, C. Blumenstein, S. Meyer, M. Wisniewski, and R. Claessen, Phys. Rev. Lett. 101, 236802 (2008).

${ }^{6}$ S. Tomonaga, Prog. Theor. Phys. 5, 544 (1950).

${ }^{7}$ J. M. Luttinger, J. Math. Phys. 4, 1154 (1963).

${ }^{8}$ F. D. M. Haldane, Phys. Rev. Lett. 47, 1840 (1981).

${ }^{9}$ I. G. Hill and A. B. McLean, Phys. Rev. B 56, 15725 (1997).

${ }^{10}$ J. R. Ahn, P. G. Kang, K. D. Ryang, and H. W. Yeom, Phys. Rev. Lett. 95, 196402 (2005).

${ }^{11}$ J. Wang, M. Li, and E. I. Altman, Phys. Rev. B 70, 233312 (2004); Surf. Sci. 596, 126 (2005).

${ }^{12}$ A. van Houselt, M. Fischer, B. Poelsema, and H. J. W. Zandvliet, Phys. Rev. B 78, 233410 (2008).

${ }^{13}$ R. Koch et al., Appl. Phys. A: Mater. Sci. Process. 55, 417 (1992).

${ }^{14}$ J. J. Schulz, M. Sturmat, and R. Koch, Phys. Rev. B 62, 15402 (2000).

${ }^{15}$ O. Gurlu et al., Appl. Phys. Lett. 83, 4610 (2003).

${ }^{16}$ N. Oncel, A. van Houselt, J. Huijben, A. S. Hallback, O. Gurlu, H. J. W. Zandvliet, and B. Poelsema, Phys. Rev. Lett. 95, 116801 (2005).

${ }^{17}$ U. Schwingenschlögl and C. Schuster, EPL 81, 26001 (2008); Eur. Phys. J. B 60, 409 (2007).

${ }^{18}$ A. A. Stekolnikov, F. Bechstedt, M. Wisniewski, J. Schafer, and R. Claessen, Phys. Rev. Lett. 100, 196101 (2008).

${ }^{19}$ P. Hohenberg and W. Kohn, Phys. Rev. 136, B864 (1964); W. Kohn and L. J. Sham, Phys. Rev. 140, A1133 (1965).

${ }^{20}$ H. Kim and J. R. Chelikowsky, Phys. Rev. Lett. 77, 1063 (1996).

${ }^{21}$ P. E. Blöchl, Phys. Rev. B 50, 17953 (1994).

${ }^{22}$ G. Kresse and D. Joubert, Phys. Rev. B 59, 1758 (1999).

${ }^{23}$ G. Kresse and J. Hafner, Phys. Rev. B 47, 558 (1993); 49, 14251 (1994).

${ }^{24}$ G. Kresse and J. Furthmüller, Comput. Mater. Sci. 6, 15 (1996); Phys. Rev. B 54, 11169 (1996).

${ }^{25}$ J. P. Perdew, K. Burke, and M. Ernzerhof, Phys. Rev. Lett. 77, 3865 (1996); 78, 1396 (1997).

${ }^{26}$ P. Pyykko, Angew. Chem., Int. Ed. 43, 4412 (2004); A. Castro et al., J. Chem. Phys. 129, 144110 (2008).

${ }^{27}$ H. J. Monkhorst and J. D. Pack, Phys. Rev. B 13, 5188 (1976).

${ }^{28}$ P. Krüger and J. Pollmann, Phys. Rev. Lett. 74, 1155 (1995).

${ }^{29}$ H. J. W. Zandvliet, Phys. Rep. 388, 1 (2003).

${ }^{30} \mathrm{~S}$. Ferrer et al., Phys. Rev. Lett. 75, 1771 (1995).

${ }^{31}$ Y. Yoshimoto et al., Phys. Rev. B 61, 1965 (2000).

${ }^{32}$ U. Schwingenschlögl and C. Schuster, Chem. Phys. Lett. 449, 126 (2007).

${ }^{33}$ A. A. Stekolnikov, J. Furthmüller, and F. Bechstedt, Phys. Rev. B 78, 155434 (2008). 